International Journal of Pure and Applied Mathematics

Volume 110 No. 3 2016, 537-545

ISSN: 1311-8080 (printed version); ISSN: 1314-3395 (on-line version)

url: http://www.ijpam.eu

doi: 10.12732/ijpam.v110i3.12

\title{
ON $w_{\tau}$-GENERALIZED CLOSED SETS IN ASSOCIATED $w$-SPACES WITH TOPOLOGIES
}

\author{
Won Keun Min ${ }^{1 \S}$, Young Key Kim² \\ ${ }^{1}$ Department of Mathematics \\ Kangwon National University \\ Chuncheon, 200-701, KOREA \\ ${ }^{2}$ Department of Mathematics \\ MyongJi University \\ Yongin, 449-728, KOREA
}

\begin{abstract}
The purpose of this paper is to introduce the notion of $w_{\tau}$-generalized closed (simply, $w_{\tau} g$-closed) sets in an associated $w_{\tau}$-space with a topology, and to study its properties. In particular, we find the following results: (1) If $f$ is $W$-continuous and closed, then for every $w_{\tau} g$-open subset $B$ in $Y, f^{-1}(B)$ is $w_{\tau} g$-open: (2) If $f$ is continuous and quasi- $W^{*}$-closed, for every $w_{\tau} g$-closed set $A$ in $X, f(A)$ is $w_{\tau} g$-closed.
\end{abstract}

AMS Subject Classification: 54A05, 54B10, 54C10, 54D30

Key Words: associated $w_{\tau}$-space, $w_{\tau}$-closed, $g$-closed, $g w_{\tau}$-closed, $w_{\tau} g$-closed

\section{Introduction}

Siwiec [13] introduced the notions of weak neighborhoods and weak base in a topological space. We introduced the weak neighborhood systems defined by using the notion of weak neighborhoods in [8]. The weak neighborhood system induces a weak neighborhood space which is independent of neighborhood spaces [2] and general topological spaces [1]. The notions of weak structure, $w$ space, $W$-continuity and $W^{*}$-continuity were investigated in [9]. In fact, the set of all $g$-closed subsets [3] in a topological space is a kind of weak structure. In

Received: $\quad$ September 23, 2016

Revised: $\quad$ October 8, 2016

Published: November 5, 2016

(c) 2016 Academic Publications, Ltd.

url: www.acadpubl.eu

$\S_{\text {Correspondence author }}$ 
[10], we introduced the notion of an associated weak space (simply, associated $w_{\tau^{-}}$-space) containing a given topology $\tau$, and moreover, we studied the notion of generalized $w_{\tau^{-}}$closed sets (simply, $g w_{\tau^{-}}$closed sets) [11] in an associated $w_{\tau^{-}}$ space with a topology $\tau$. Now, the one purpose of this research is to study $w_{\tau}$-generalize closed sets in an associated weak space $w_{\tau}$ in the similar way introduced by Levine [3] in topological spaces. So we introduce the notion of $w_{\tau}$-generalized closed (simply, $w_{\tau} g$-closed) sets in an associated $w_{\tau}$-space with a topology, and to study its properties. In particular, we find the following results: (1) If $f$ is $W$-continuous and closed, then for every $w_{\tau} g$-open subset $B$ in $Y, f^{-1}(B)$ is $w_{\tau} g$-open: (2) if $f$ is continuous and quasi- $W^{*}$-closed, for every $w_{\tau} g$-closed set $A$ in $X, f(A)$ is $w_{\tau} g$-closed.

\section{Preliminaries}

Definition $2.1([9])$. Let $X$ be a nonempty set. A subfamily $w_{X}$ of the power set $P(X)$ is called a weak structure on $X$ if it satisfies the following:

(1) $\emptyset \in w_{X}$ and $X \in w_{X}$.

(2) For $U_{1}, U_{2} \in w_{X}, U_{1} \cap U_{2} \in w_{X}$.

Then the pair $\left(X, w_{X}\right)$ is called a $w$-space on $X$. Then $V \in w_{X}$ is called a $w$-open set and the complement of a $w$-open set is a $w$-closed set.

The collection of all $w$-open sets (resp., $w$-closed sets) in a $w$-space $X$ will be denoted by $W O(X)$ (resp., $W C(X))$. We set $W(x)=\{U \in W O(X): x \in U\}$.

Let $S$ be a subset of a topological space $X$. The closure (resp., interior) of $S$ will be denoted by $c l S$ (resp., int $S$ ). A subset $S$ of $X$ is called a preopen set [6] (resp., $\alpha$-open set [12], semi-open [4]) if $S \subset \operatorname{int}(\operatorname{cl}(S))$ (resp., $S \subset$ $\operatorname{int}(\operatorname{cl}(\operatorname{int}(S))), S \subset \operatorname{cl}(\operatorname{int}(S)))$. The complement of a preopen set (resp., $\alpha-$ open set, semi-open) is called a preclosed set (resp., $\alpha$-closed set, semi-closed). The family of all preopen sets (resp., $\alpha$-open sets, semi-open sets) in $X$ will be denoted by $P O(X)$ (resp., $\alpha(X), S O(X)$ ). We know the family $\alpha(X)$ is a topology finer than the given topology on $X$.

Moreover, a subset $S$ of $X$ is said to be $g$-closed [3] if $\operatorname{cl}(A) \subset U$ whenever $A \subset U$ and $U$ is open in $X$.

Then the family $G O(X)=\{U \subseteq X: U$ is $g$-open $\}, O(X)=\{U \subseteq X$ : $U$ is open $\}$ and $C L(X)=\{F \subseteq X: F$ is closed $\}$ are all weak structures on $X$. But $P O(X), G P O(X)$ and $S O(X)$ are not weak structures on $X$. A subfamily $m_{X}$ of the power set $P(X)$ of a nonempty set $X$ is called a minimal 
structure on $X[5]$ if $\emptyset \in w_{X}$ and $X \in w_{X}$. Thus clearly every weak structure is a minimal structure.

Let $\left(X, w_{X}\right)$ be a $w$-space. For a subset $A$ of $X$, the $w$-closure of $A$ and the $w$-interior of $A$ are defined as follows:

(1) $w C(A)=\cap\left\{F: A \subseteq F, X-F \in w_{X}\right\}$.

(2) $w I(A)=\cup\left\{U: U \subseteq A, U \in w_{X}\right\}$.

Theorem $2.2([9])$. Let $\left(X, w_{X}\right)$ be a $w$-space and $A \subseteq X$. Then the following things hold:

(1) If $A \subseteq B$, then $w I(A) \subseteq w I(B) ; w C(A) \subseteq w C(B)$.

(2) $w I(w I(A))=w I(A) ; w C(w C(A))=w C(A)$.

(3) $w C(X-A)=X-w I(A) ; w I(X-A)=X-w C(A)$.

(4) If $A$ is $w$-closed (resp., $w$-open), then $w C(A)=A$ (resp., $w I(A)=A$ ).

Let $X$ be a nonempty set and let $(X, \tau)$ be a topological space. A subfamily $w$ of the power set $P(X)$ is called an associated weak structure (simply, $w_{\tau}$ ) [10] on $X$ if $\tau \subseteq w$ and $w$ is a weak structure. Then the pair $\left(X, w_{\tau}\right)$ is called an associated $w$-space with $\tau$.

\section{Main Results}

Definition 3.1. Let $\left(X, w_{\tau}\right)$ be an associated $w$-space with a topology $\tau$ and $A \subseteq X$. Then $A$ is called a $w_{\tau}$-generalized closed set (simply, $w_{\tau} g$-closed set) if $w C(A) \subseteq U$, whenever $A \subseteq U$ and $U$ is open.

We recall that: $A$ is called a generalized closed set (simply, g-closed set) [3] if $\operatorname{cl}(A) \subseteq U$, whenever $A \subseteq U$ and $U$ is open.

Remark 3.2. (1) If $w_{\tau}=\tau$, then the $w_{\tau}$-generalized closed set is exactly a $g$-closed set.

(2) If $w_{\tau}$ is the family of all $g$-open sets in sense of Levine, then the generalized $w_{\tau}$-closed set is exactly a $g^{*}$-closed set [14].

Theorem 3.3. Every $g$-closed set is $w_{\tau}$-generalized closed.

Proof. Since $w C(A) \subseteq \operatorname{cl}(A)$, every $g w_{\tau}$-closed set is $w_{\tau} g$-closed.

In general, the converse of the above theorem is not true as the next example. 
Example 3.4. Let $X=\{a, b, c, d\}$, a topology $\tau=\{\emptyset,\{b\},\{b, c\}, X\}$ and a $w$-structure $w_{X}=\{\emptyset,\{a\},\{b\},\{b, c\},\{a, d\}, X\}$ in $X$. For $A=\{c\}$, since $w C(A)=A$ and $c l(A)=\{a, c, d\}$, obviously $A$ is $w_{\tau} g$-closed but not $g$-closed.

Let $\left(X, w_{\tau}\right)$ be an associated $w$-space with a topology $\tau$ and $A \subseteq X$. Then $A$ is called a generalized $w_{\tau}$-closed set (simply, $g w_{\tau}$-closed set) [11] if $\operatorname{cl}(A) \subseteq U$, whenever $A \subseteq U$ and $U$ is $w$-open. Then since $\tau \subseteq w$, every $g w_{\tau}$-closed set is $w_{\tau} g$-closed. But, the converse may not be true as the next example.

Example 3.5. In Example 3.3, let $A=\{a\}$. Then $w C(A)=\{a, d\}$, and clearly $A$ is $w_{\tau} g$-closed. Now, consider a $w$-open set $U=\{a\}$ such that $A \subseteq U$. Then $A$ is not $g w_{\tau}$-closed.

For a given associated $w_{\tau}$-space with a topology $\tau$, from the above theorems and examples, the following relations are obtained:

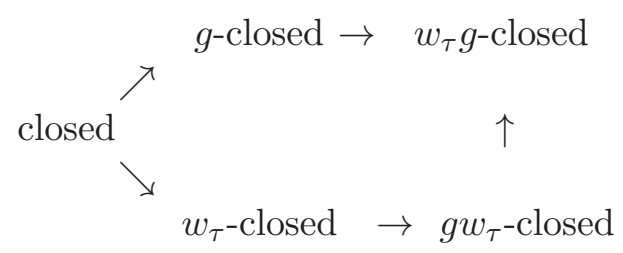

Lemma $3.6([9])$. Let $\left(X, w_{\tau}\right)$ be an associated $w$-space with a topology $\tau$ and $A, B \subseteq X$. Then the following things hold:

(1) $w I(A) \cap w I(B)=w I(A \cap B)$.

(2) $w C(A) \cup w C(B)=w C(A \cup B)$.

Theorem 3.7. Let $\left(X, w_{\tau}\right)$ be an associated $w$-space with a topology $\tau$. Then the union of two $w_{\tau} g$-closed sets is a $w_{\tau} g$-closed set.

Proof. Let $A$ and $B$ be any two $w_{\tau} g$-closed sets. Let $G$ be any open set such that $A \cup B \subseteq G$. Then $A \subseteq G$ and $B \subseteq G$. Since $A$ and $B$ are $w_{\tau} g$-closed sets, $w C(A) \subseteq G$ and $w C(B) \subseteq G$. Now, by the above lemma, $w C(A \cup B) \subseteq G$, and so $A \cup B$ is $w_{\tau} g$-closed.

In general, the intersection of two $w_{\tau} g$-closed sets is not $w_{\tau} g$-closed:

Example 3.8. Let $X=\{a, b, c, d\}$, a topology $\tau=\{\emptyset,\{a, c\}, X\}$ and a $w$-structure $w_{X}=\{\emptyset,\{a, c\},\{a\},\{b\},\{c\},\{a, d\}, X\}$ in $X$. Now, consider $A=\{a, b, c\}$ and $B=\{a, c, d\}$. Then $A$ and $B$ are $w_{\tau} g$-closed. But for $A \cap B=\{a, c\}$, since $\{a, c\}$ is open and $w C(\{a, c\})=\{a, c, d\}$, the intersection is not $w_{\tau} g$-closed. 
Theorem 3.9. Let $\left(X, w_{\tau}\right)$ be an associated $w$-space with a topology $\tau$. Then if $A$ is a $w_{\tau} g$-closed set, then $w C(A)-A$ contains no non-empty closed set.

Proof. Let $F$ be a closed set such that $F \subseteq w C(A)-A$. Then $A \subseteq X-F$. Since $A$ is $w_{\tau} g$-closed, $w C(A) \subseteq X-F$. It implies that $F \subseteq w C(A) \cap(X-$ $w C(A))=\emptyset$. So, $F=\emptyset$.

In general, the converse in Theorem 3.9 is not true as shown in the next example.

Example 3.10. Let $X=\{a, b, c, d\}$, a topology $\tau=\{\emptyset,\{a\},\{a, d\}, X\}$ and $w_{X}=\{\emptyset,\{a\},\{b\},\{c\},\{a, d\}, X\}$ be a $w$-structure in $X$. Consider $A=\{a\}$. Note $w C(A)=\{a, d\}$ and $w C(A)-A=\{a, d\}-\{a\}=\{d\}$. Since $\{d\}$ is not closed, $w C(A)-A$ contains no non-empty closed set, but $A$ is not $w_{\tau} g$-closed.

Theorem 3.11. Let $\left(X, w_{\tau}\right)$ be an associated $w$-space with a topology $\tau$. Then if $A$ is a $w_{\tau} g$-closed set and $A \subseteq B \subseteq w C(A)$, then $B$ is $w_{\tau} g$-closed.

Proof. Let $U$ be any open set such that $B \subseteq U$. Since $A$ is a $w_{\tau} g$-closed set and $w C(B) \subseteq w C(A), w C(B) \subseteq U$, and so $B$ is $w_{\tau} g$-closed set.

Definition 3.12. Let $\left(X, w_{\tau}\right)$ be an associated $w$-space with a topology $\tau$ and $A \subseteq X$. Then $A$ is called a $w_{\tau}$-generalized open set (simply, $w_{\tau} g$-open set) if $X-A$ is $w_{\tau} g$-closed.

Theorem 3.13. Let $\left(X, w_{\tau}\right)$ be an associated $w$-space with a topology $\tau$ and $A \subseteq X$. Then $A$ is $w_{\tau} g$-open if and only if $F \subseteq w I(A)$ whenever $F \subseteq A$ and $F$ is closed.

Proof. From Definition 3.1, it is obvious.

Theorem 3.14. Let $\left(X, w_{\tau}\right)$ be an associated $w$-space with a topology $\tau$. Then the intersection of two $w_{\tau} g$-open sets is a $w_{\tau} g$-open set.

Proof. It is obvious from Theorem 3.7 and Theorem 2.2.

In general, the union of two $w_{\tau} g$-open sets is not $w_{\tau} g$-open (See Example $3.8)$.

Theorem 3.15. Let $\left(X, w_{\tau}\right)$ be an associated $w$-space with a topology $\tau$ and $A \subseteq X$. Then if $A$ is $w_{\tau} g$-open, then $U=X$, whenever $w I(A) \cup(X-A) \subseteq U$ and $U$ is open. 
Proof. For $w_{\tau} g$-open set $A$, let $U$ be any open set satisfying $w I(A) \cup(X-$ $A) \subseteq U$. Then $X-U \subseteq(X-w I(A)) \cap A=w C(X-A) \cap A=w C(X-A)-$ $(X-A)$. Since $X-A$ is $w_{\tau} g$-closed, by Theorem 3.9, $X-U$ must be empty. Thus, $U=X$.

Theorem 3.16. Let $\left(X, w_{\tau}\right)$ be an associated $w$-space with a topology $\tau$. Then if $A$ is a $w_{\tau} g$-open set and $w I(A) \subseteq B \subseteq A$, then $B$ is $w_{\tau} g$-open.

Proof. It is similar to the proof of Theorem 3.11.

Theorem 3.17. Let $\left(X, w_{\tau}\right)$ be an associated $w$-space with a topology $\tau$. Then if $A$ is a $w_{\tau} g$-closed set, then $w C(A)-A$ is $w_{\tau} g$-open.

Proof. If $A$ is a $w_{\tau} g$-closed set, then by Theorem 3.11 , the empty set is the only one closed subset of $w C(A)-A$. So, for the only closed subset $\emptyset$ of $w C(A)-A, \emptyset \subseteq w C(A)-A$ and $\emptyset \subseteq w I(w C(A)-A)$. Hence, $w C(A)-A$ is $w_{\tau} g$-open.

Theorem 3.18. Let $\left(X, w_{\tau}\right)$ be an associated $w$-space with a topology $\tau$. Then if $A$ is a $w_{\tau} g$-open set, then $w I(A) \cup(X-A)$ is $w_{\tau} g$-closed.

Proof. If $A$ is a $w_{\tau} g$-open set, then by Theorem $3.15, X$ is the only one open set containing $w I(A) \cup(X-A)$. So, $w I(A) \cup(X-A) \subseteq X$ and $w C(w I(A) \cup$ $(X-A)) \subseteq X$. Hence, $w I(A) \cup(X-A)$ is $w_{\tau} g$-closed.

Let $\left(X, w_{\tau}\right)$ be an associated $w$-space with a topology $\tau$. For a subset $A$ of $X, w_{\tau} g$-closure of $A$ and $w_{\tau} g$-interior of $A$ are defined as the following:

(1) $w_{\tau} g C(A)=\cap\left\{F: A \subseteq F, F\right.$ is $w_{\tau} g$-closed $\}$.

(2) $w_{\tau} g I(A)=\cup\left\{U: U \subseteq A, U\right.$ is $w_{\tau} g$-open $\}$.

Theorem 3.19. Let $\left(X, w_{\tau}\right)$ be an associated $w$-space with a topology $\tau$ and $A \subseteq X$.

(1) If $A$ is $w_{\tau} g$-open, then $w_{\tau} g I(A)=A$.

(2) If $A$ is $w_{\tau} g$-closed, then $w_{\tau} g C(A)=A$.

Proof. Obvious.

But the converses in the above theorem are not always true as shown in the next example. 
Example 3.20. In Example 3.8, let $F=\{a, c\}$. Since $\{a, b, c\}$ and $\{a, c, d\}$ are $w_{\tau} g$-closed sets, $w_{\tau} g C(F)=\{a, c\}$. But $F$ is not $w_{\tau} g$-closed. Similarly, we can show that the converse of (2) in Theorem 3.19 may not be true.

The following things are easily obtained by the notions of $w_{\tau} g$-closure and $w_{\tau} g$-interior:

Theorem 3.21. Let $\left(X, w_{\tau}\right)$ be an associated $w$-space with a topology $\tau$ and $A, B \subseteq X$.

(1) If $A \subseteq B$, then $w_{\tau} g I(A) \subseteq w_{\tau} g I(B)$ and $w_{\tau} g C(A) \subseteq w_{\tau} g C(B)$.

(2) $w_{\tau} g C(X-A)=X-w_{\tau} g I(A) ; w_{\tau} g I(X-A)=X-w_{\tau} g C(A)$.

(3) $x \in w_{\tau} g I(A)$ if and only if there exists a $w_{\tau} g$-open set $U$ containing $x$ such that $U \subseteq A$.

(4) $x \in w_{\tau} g C(A)$ if and only if $A \cap V \neq \emptyset$ for all $w_{\tau} g$-open set $V$ containing $x$.

Theorem 3.22. Let $\left(X, w_{\tau}\right)$ be an associated $w$-space with a topology $\tau$ and $A, B \subset X$.

(1) $\emptyset=w_{\tau} g C(\emptyset)$.

(2) $A \subseteq w_{\tau} g C(A)$.

(3) $w_{\tau} g C(A \cup B)=w_{\tau} g C(A) \cup w_{\tau} g C(B)$.

(4) $w_{\tau} g C\left(w_{\tau} g C(A)\right)=w_{\tau} g C(A)$.

Proof. (1) and (2) are obvious.

(3) Now, we only show that $w_{\tau} g C(A \cup B) \subseteq w_{\tau} g C(A) \cup w_{\tau} g C(B)$. For the proof, let $x \notin w_{\tau} g C(A) \cup w_{\tau} g C(B)$. Then there exist $w_{\tau} g$-closed sets $F_{1}$ and $F_{2}$ such that $x \notin F_{1}$ and $A \subseteq F_{1} ; x \notin F_{2}$ and $B \subseteq F_{2}$. So $x \notin F_{1} \cup F_{2}$ and $A \cup B \subseteq F_{1} \cup F_{2}$. Since $F_{1} \cup F_{2}$ is $w_{\tau} g$-closed, we have that $x \notin w_{\tau} g C(A \cup B)$. Hence, $w_{\tau} g C(A \cup B) \subseteq w_{\tau} g C(A) \cup w_{\tau} g C(B)$.

(4) It is clear that $w_{\tau} g C\left(w_{\tau} g C(A)\right) \supseteq w_{\tau} g C(A)$. Now, we show that $w_{\tau} g C\left(w_{\tau} g C(A)\right) \subseteq w_{\tau} g C(A)$. First, for any $w_{\tau} g$-closed set $F$ satisfying $A \subseteq F$, we know that $w_{\tau} g C(A) \subseteq w_{\tau} g C(F)=F$. So, from this fact, $\{F: A \subseteq$ $F, F$ is $w_{\tau} g$-closed $\} \subseteq\left\{K: w_{\tau} g C(A) \subseteq K, K\right.$ is $w_{\tau} g$-closed $\}$. This implies that $w_{\tau} g C\left(w_{\tau} g C(A)\right)=\cap\left\{K: w_{\tau} g C(A) \subseteq K, K\right.$ is $w_{\tau} g$-closed $\} \subseteq \cap\{F: A \subseteq$ $F, F$ is $w_{\tau} g$-closed $\}=w_{\tau} g C(A)$.

From Theorem 3.21 and Theorem 3.22, the following are obtained: 
Theorem 3.23. Let $\left(X, w_{\tau}\right)$ be an associated $w$-space with a topology $\tau$ and $A, B \subseteq X$.

(1) $X=w_{\tau} g I(X)$.

(2) $w_{\tau} g I(A) \subseteq A$.

(3) $w_{\tau} g I(A \cap B)=w_{\tau} g I(A) \cap w_{\tau} g I(B)$.

(4) $w_{\tau} g I\left(w_{\tau} g I(A)\right)=w_{\tau} g I(A)$.

Finally, from Theorem 3.3 and Theorem 3.23, we have a topology induced by $w_{\tau} g$-open sets as the following:

Theorem 3.24. Let $\left(X, w_{\tau}\right)$ be an associated $w$-space with a topology $\tau$. Then the family $\tau^{*}=\left\{U \subseteq X: U=w_{\tau} g I(U)\right\}$ is a topology such that $\tau \subseteq w_{\tau} \subseteq \tau^{*}$ and $G O(X) \subseteq \tau^{*}$.

Recall the notion of $W$-continuity: Let $\left(X, w_{X}\right)$ and $\left(Y, w_{Y}\right)$ be two $w$ spaces. Then $f: X \rightarrow Y$ is said to be:

(1) $W$-continuous [9] if for $x \in X$ and $V \in W(f(x))$, there is $U \in W(x)$ such that $f(U) \subseteq V$;

(2) $W^{*}$-closed [11] if for every $w$-closed set $F$ in $X, f(F)$ is a $w$-closed set in $Y$.

(3) quasi- $W^{*}$-closed [11] if for $A \subseteq X, w C(f(A)) \subseteq f(w C(A))$.

In fact, there is no any relation between the notions of $W^{*}$-closed function is quasi- $W^{*}$-closed function.

Theorem $3.25([9])$. Let $f:\left(X, w_{X}\right) \rightarrow\left(Y, w_{Y}\right)$ be a function in two $w$-spaces. Then the following statements are equivalent:

(1) $f$ is $W$-continuous.

(2) $f(w C(A)) \subseteq w C(f(A))$ for $A \subseteq X$.

(3) $w C\left(f^{-1}(B)\right) \subseteq f^{-1}(w C(B))$ for $B \subseteq Y$.

(4) $f^{-1}(w I(B)) \subseteq w I\left(f^{-1}(B)\right)$ for $B \subseteq Y$.

Theorem 3.26. Let $f:\left(X, w_{\tau_{X}}\right) \rightarrow\left(Y, w_{\tau_{Y}}\right)$ be a function on associated $w$-spaces $X$ and $Y$. Then if $f$ is $W$-continuous and closed, then for every $w_{\tau} g$-open subset $B$ in $Y, f^{-1}(B)$ is $w_{\tau} g$-open.

Proof. Let $B$ be any $w_{\tau} g$-open subset in $Y$ and $F$ be a closed set in $X$ such that $F \subseteq f^{-1}(B)$. Now, we show that $F \subseteq w I\left(f^{-1}(B)\right)$. Since $B$ is $w_{\tau} g$-open and $f(F)$ is closed, $f(F) \subseteq w I(B)$. From (4) of Theorem 3.25, it follows that $F \subseteq f^{-1}(w I(B)) \subseteq w I\left(f^{-1}(B)\right)$. Hence, $f^{-1}(B)$ is $w_{\tau} g$-open. 
Theorem 3.27. Let $f:\left(X, w_{\tau_{X}}\right) \rightarrow\left(Y, w_{\tau_{Y}}\right)$ be a function on associated $w$-spaces $X$ and $Y$. Then if $f$ is continuous and quasi- $W^{*}$-closed, for every $w_{\tau} g$-closed set $A$ in $X, f(A)$ is $w_{\tau} g$-closed.

Proof. For a $w_{\tau} g$-closed subset $A$ in $X$, let $U$ be any open set in $Y$ such that $f(A) \subseteq U$. Now, we show that $w C(f(A)) \subseteq U$. Since $f$ is continuous and $A$ is $w_{\tau} g$-closed, $f^{-1}(U)$ is open and $w C(A) \subseteq f^{-1}(U)$. Since $f$ is quasi- $W^{*}$-closed, $w C(f(A)) \subseteq f(w C(A)) \subseteq f f^{-1}(U) \subseteq U$. Hence, $f(A)$ is $w_{\tau} g$-closed.

\section{References}

[1] Á. Csázár, Generalized topology, generalized continuity, Acta Math. Hungar., 96 (2002), 351-357.

[2] D.C. Kent and W.K. Min, Neighborhood spaces, International Journal of Mathematics and Mathematical Sciences, 32, No. 7 (2002), 387-399.

[3] N. Levine, Generalized closed sets in topology, Rend. Cir. Mat. Palermo, 19 (1970), 89-96.

[4] N. Levine, Semi-open sets and semi-continuity in topological spaces, Ams. Math. Monthly, 70 (1963), 36-41.

[5] H. Maki, On generalizing semi-open and preopen sets, Report for Meeting on Topological Spaces Theory and its Applications, August 1996, Yatsushiro College of Technology, 1318.

[6] A.S. Mashhour, M.E. Abd El-Monsef and S.N. El-Deeb, On precontinuous and weak precontinuous mappings, Proc. Math. and Phys. Soc. Egypt, 53 (1982), 47-53.

[7] W.K. Min, Some results on generalized topological spaces and generalized systems, Acta Math. Hungar., 108, No-s: 1-2 (2005), 171-181.

[8] W.K. Min, On weak neighborhood systems and spaces, Acta Math. Hungar., 121, No. 3 (2008), 283-292.

[9] W.K. Min, Y.K. Kim, On weak structures and w-spaces, Far East Journal of Mathematical Sciences, 97, No. 5 (2015), 549-561.

[10] W.K. Min and Y.K. Kim, $W O$-continuity and $W K$-continuity On Associated $w$-spaces, International Journal of Pure and Applied Mathematics, 102, No. 2 (2015), 349-356.

[11] W.K. Min and Y.K. Kim, On generalized $w$-closed Sets in associated weak spaces, Submitted.

[12] O. Njastad, On some classes of nearly open sets, Pacific Journal of Mathematics, 15, No. 3 (1964), 961-970.

[13] F. Siwiec, On defining a space by a weak base, Pacific Journal of Mathematics, 52, No. 1 (1974), 351-357.

[14] M.K.R.S. Veerakumar, Between closed sets and g-closed sets, Mem. Fac. Sci. Kochi. Univ. Ser. A: Math., 17, No. 21 (2000), 1-19. 
